\title{
THE RADON TRANSFORM ON A FAMILY OF CURVES IN THE PLANE. II
}

\author{
A. M. CORMACK
}

\begin{abstract}
A further discussion of Radon's problem for curves in the plane given, for fixed $(p, \phi)$, by $r^{\alpha} \cos \{\alpha(\theta-\phi)\}=p^{\alpha}, \alpha$ real, $\alpha \neq 0$. Functions yielding null transforms, and zeros of the Fourier components of the transforms are given for general $\alpha$, and several orthogonal expansions are given for $\alpha= \pm 1 / m, m=$ $1,2,3 \ldots$
\end{abstract}

1. Introduction. If $f$ is a function in the plane and $\hat{f}$ represents the integral of $f$ along a straight line in the plane, the problem of recovering $f$ from all the $\hat{f}$ was originally solved by Radon, and $\hat{f}$ will be called the Radon transform of $f$. The problem has been generalized in many ways $[6,5]$, and recently $[4]$ formulae were given for recovering $f$ from $\hat{f}$ where the integrals were performed on the following curves in the plane. Let $(r, \theta)$ be polar coordinates to a point on the curve specified by polar parameters $(p, \phi)$, then the equation of one family of curves, called the $\alpha$-curves, is

$$
r^{\alpha} \cos \{\alpha(\theta-\phi)\}=p^{\alpha}, \quad \alpha>0,|\theta-\phi|<\pi / 2 \alpha,
$$

and the other, called the $\beta$-curves, is

$$
p^{\beta} \cos \{\beta(\theta-\phi)\}=r^{\beta}, \quad \beta>0,|\theta-\phi|<\pi / 2 \beta .
$$

For $\alpha=\beta$ an $\alpha$-curve is an inversion in the unit circle $((r, \theta) \rightarrow(1 / r, \theta))$ of a $\beta$-curve, and conversely. Because they have somewhat different properties, it is convenient to treat the two families separately, rather than as a single family with, say, $-\infty<\alpha<\infty, \alpha \neq 0$. The function $f$ will be considered to be smooth in all cases. It will variously be assumed to fall off faster than $(1 / r)$, or to be rapidly decreasing, or to be of compact support. The Radon transforms are

$$
\hat{f}(p, \phi)=\int_{\alpha} f(r, \theta) d s_{\alpha}, \quad \hat{f}(p, \theta)=\int_{\beta} f(r, \theta) d s_{\beta}
$$

where $d s_{\alpha}$ and $d s_{\beta}$ are elements of length of an $\alpha$-curve or a $\beta$-curve, respectively.

The following results were given in [4]. If $f$ and $\hat{f}$ are expanded in Fourier series,

$$
f(r, \theta)=\sum_{l=-\infty}^{+\infty} f_{l}(r) e^{i l \theta}, \quad \hat{f}(p, \theta)=\sum_{l=-\infty}^{+\infty} \hat{f}_{l}(p) e^{i l \phi},
$$

Received by the editors February 1, 1982; presented in part at the Amer. Math. Soc. Short Course on Computed Tomography, Cincinnati, January 11, 1982.

1980 Mathematics Subject Classification. Primary 44A05, 45A05.

Key words and phrases. Radon transform. 
then $f_{l}$ and $\hat{f}_{l}$ are connected by an integral equation, but that this could be reduced to a simpler form, reminiscent of the expansion of the ordinary Radon transform in Tschebycheff polynomials, by writing

$$
\begin{aligned}
& F_{l}(s)=\frac{1}{\alpha} f_{l}\left(s^{1 / \alpha}\right) s^{1 / \alpha-1}, \\
& \hat{F}_{l}(q)=\hat{f}_{l}\left(q^{1 / \alpha}\right),
\end{aligned}
$$

with the same relations for the $\beta$-curves. The $F_{l}$ and $\hat{F}_{l}$ are related as follows. For the $\alpha$-curves

$$
\begin{aligned}
\hat{F}_{l}(q) & =2 \int_{q}^{\infty} F_{l}(s) \frac{\cos \left\{(l / \alpha) \cos ^{-1}(q / s)\right\} d s}{\left(1-(q / s)^{2}\right)^{1 / 2}} \\
& =2 q \int_{0}^{\pi / 2} F_{l}(q \sec \theta) \cos \{(l / \alpha) \theta\} \cos ^{-2} \theta d \theta,
\end{aligned}
$$

and for the $\beta$-curves

$$
\begin{aligned}
\hat{F}_{l}(q) & =2 \int_{0}^{q} F_{l}(s) \frac{\cos \left\{(l / \beta) \cos ^{-1}(s / q)\right\} d s}{\left(1-(s / q)^{2}\right)^{1 / 2}} \\
& =2 q \int_{0}^{\pi / 2} F_{l}(q \cos \theta) \cos \{(l / \beta) \theta\} d \theta
\end{aligned}
$$

Inversion formulas were given in [4] but they are not needed here.

After a discussion of functions in the null-space of $\hat{F}$, and of the zeros of $\hat{F}_{l}$, for general values of $\alpha$ and $\beta$, a number of results will be given for the special cases $\alpha$ or $\beta=1 / m, m=1,2,3, \ldots$. The case $\alpha=1$ corresponds to the ordinary Radon transform which has been extensively written about, and the case $\beta=1$ corresponds to circles through the origin which have been discussed in [3]. The results to be given for these special cases are all similar to known results, so proofs will only be sketched and the reader will be referred to the original papers for details.

The following integral will be used frequently $[8, \mathrm{p}$. 9]. When $R l \nu>0$

$$
\begin{aligned}
I(\nu, a) & =\int_{0}^{\pi / 2} \cos ^{\nu-1} \theta \cos a \theta d \theta \\
& =\frac{\pi}{\nu 2^{\nu}} \frac{\Gamma(\nu+1)}{\Gamma((1+\nu+a) / 2) \Gamma((1+\nu-a) / 2)} .
\end{aligned}
$$

We will use this only for real values of $\nu . \Gamma(z)$ is the gamma-function which is analytic except for simple poles at $z=0,-1,-2, \ldots$, and it is positive on the real axis. If $a>0$ the poles of the second gamma-function in the denominator cause $I$ to vanish, so

$$
I(\nu, a)=0 \text { for } \nu=a-1, a-3, \ldots, a-(2 k+1)
$$

where $k(=0,1,2 \ldots)$ is the largest integer satisfying $k<\frac{1}{2}(a-1)$.

2. Null transforms and zeros of $\hat{F}_{l}$. It is well known that the ordinary Radon transform is zero for $p>0$ if $f_{l}(r)=(1 / r)^{k+2}, r>0$, and $l-k$ is even, $k<|l|[10]$, so it is natural to put $F_{l}(s)=1 / s^{\gamma}, s>0$, or $s^{\gamma}, \gamma>0$. Putting these into (7) or (8) 
and applying (10) immediately yields the following results. If for a given $l(\neq 0)$ and $\alpha($ or $\beta)$ there is a $k$ such that $X$ then $\hat{F}_{l}(q)=0$ for $\gamma=Y$, where $X$ is a restriction on $k$ and $Y$ is a set of values of $\gamma$ given in the following table:

$\begin{array}{cccc} & F_{l} & X & Y \\ \alpha \text {-curves } & s^{-\gamma} & k<\frac{1}{2}\left(\frac{l}{\alpha}-1\right) & (l / \alpha),(l / \alpha)-2, \ldots,(l / \alpha)-2 k \\ \beta \text {-curves } & s^{-\gamma} & 2 k+1<\frac{l}{\beta}<2 k+2 & 2 k+2-(l / \beta) \\ \beta \text {-curves } & s^{\gamma} & k<\frac{1}{2}\left(\frac{l}{\beta}-1\right) & (l / \beta)-2,(l / \beta)-4, \ldots,(l / \beta)-(2 k+2)\end{array}$

Further argument is necessary to see whether or not the above $F_{l}$ comprise the entire null-spaces of the transforms as they do in the cases $\alpha=1$ and $\beta=1$ [11].

In [1] it was established that for the ordinary Radon transform $(\alpha=1) \hat{f}_{2 l}$ and $\hat{f}_{2 l+1}$ each had at least $l$ zeros in $[0, \infty)$ by first establishing that $\int_{0}^{\infty} \hat{f}_{l}(p) p^{k} d p$ vanished for certain values of $k$, and then considering $\int_{0}^{\infty} \hat{f}_{l}(p) \prod_{j=1}^{m}\left(p^{2}-p_{j}^{2}\right) d p$ where the $p_{j}$ were $m$ assumed zeros of $\hat{f}_{l}$. A contradiction resulted unless $m$ had at least a certain value. The same method of proof, again using (10), works for the $\alpha$-curves and yields the following result. If $l / \alpha=[l / \alpha]+\delta$, where $[x]$ denotes the largest integer less than or equal to $x$, and where $0 \leqslant \delta<1$, then $\hat{F}_{l}(q)$ has $r$ ( $r=1,2,3, \ldots$ ) zeros, not including $q=0$, in $(0, \infty)$ if $[l / \alpha]=2 r$ or $2 r+1$. If $l$ is odd there is an additional zero at $q=0$.

For the $\beta$-curves the argument follows that given in [4] for the case $\beta=1$, which is similar to the above discussion of the $\alpha$-curves except that the starting point is the consideration of $\int_{0}^{\infty} \hat{F}_{l}(q) d q / q^{\gamma}$ and the use of (10) again. The result is as follows: $\hat{F}_{l}(q)$ has at least $r+1$ zeros in $[0, \infty)$ if $[l / r]=2 r$ or $2 r+1$. If $l$ is odd there is an additional zero at $q=0$.

3. Special cases: $\alpha=1 / m, m=1,2,3, \ldots$ In these cases $\cos \left\{(l / \alpha) \cos ^{-1} x\right\}=$ $\cos \left\{\operatorname{lm} \cos ^{-1} x\right\}=T_{l m}(x)$ where $T_{k}(x)$ is the Tschebycheff polynomial of the first kind. The ordinary Radon transform is given by $m=1$, and some of the many studies of this case form the bases of the results given below. Proofs will not be given because the results are straightforward generalizations of published work.

(a)

$$
\int_{-\infty}^{+\infty} \hat{F}_{l}(q) e^{i q t} d q=2 \pi(i)^{l m} \int_{0}^{\infty} F_{l}(s) J_{l m}(s t) s d s
$$

The proof is the same as in [2].

(b) Three orthogonal expansions.

We need the four well-known sets of orthogonal functions given with their weights and intervals of orthogonality in the following table [8]:

\begin{tabular}{|c|c|c|c|}
\hline Name & Symbol & Weight Function & Interval \\
\hline$\overline{\text { Hermite }}$ & $\overline{H_{n}(x)}$ & $\overline{e^{-x^{2}}}$ & $\overline{(-\infty, \infty)}$ \\
\hline Laguerre & $L_{n}^{\alpha}(x)$ & $x^{\alpha} e^{-x}, \alpha>-1$ & $(0, \infty)$ \\
\hline Gegenbauer & $C_{n}^{\lambda}(x)$ & $\left(1-x^{2}\right)^{\lambda-1 / 2}, \lambda>-\frac{1}{2}$ & $(-1,1)$ \\
\hline Shifted Jacobi & $G_{n}(p, q, x)$ & $(1-x)^{p-q} x^{q-1}, p-q>-1, q>0$ & $(0,1)$ \\
\hline
\end{tabular}


(i) Smooth rapidly decreasing functions in $(0, \infty)$.

By applying (11) to $F_{l}(s)$ as given below, and by using exactly the same methods as in [2] one finds that if

$$
F_{l}(s)=s^{l m} e^{-s^{2}} L_{k}^{l m}\left(s^{2}\right),
$$

then

$$
\hat{F}_{l}(q)=\frac{(-1)^{l m+k} \sqrt{\pi}}{2^{l m+2 k} k !} e^{-q^{2}} H_{l m+2 k}(q) .
$$

(ii) Smooth functions of compact support in $(0,1)$.

If

$$
F_{l}(s)=s^{l m}\left(1-s^{2}\right)^{\lambda-1 / 2} G_{k}\left(\operatorname{lm}+\lambda, \operatorname{lm}+1, s^{2}\right), \quad \lambda>-\frac{1}{2}
$$

then

$$
\begin{aligned}
\hat{F}_{l}(q)= & 2^{2 \lambda-1} B(\lambda, l m+2 k) B(k+\lambda, l m+k+\lambda) \\
& \times\left(1-q^{2}\right)^{\lambda-1 / 2} C_{l m+2 k}^{\lambda}(q)
\end{aligned}
$$

where $B(x, y)=\Gamma(x) \Gamma(y) / \Gamma(x+y)$. For the ordinary Radon transform $(m=1)$ and for $\lambda=1$ this result has been extensively studied [2,9], and the extension to general $\lambda$ has been given by Louis [7]. The result given in (13a) and (13b) is obtained in exactly the same way as Louis's result.

(iii) Smooth rapidly decreasing functions in $(1, \infty)$.

If $\operatorname{lm}=2 r, r=0,1,2,3, \ldots$ and if

$$
\begin{gathered}
F_{l}(s)=\left(1 / s^{2}\right) G_{k}\left(\lambda, 1 / 2,1 / s^{2}\right), \\
\hat{F}_{l}(q)=\frac{\sqrt{\pi}}{q^{2 r+1}} \frac{(\gamma+r) !}{\gamma !} \frac{\Gamma(\gamma+r+1 / 2)}{\Gamma(\gamma+2 r+1)} G_{\gamma}\left(\lambda+2 r, 2 r+1,1 / q^{2}\right)
\end{gathered}
$$

where $\gamma=k-r$.

If $l m=2 r+1, r=0,1,2, \ldots$ and if

$$
F_{l}(s)=\left(1 / s^{3}\right) G_{k}\left(\lambda, 3 / 2,1 / s^{2}\right)
$$

$$
\hat{F}_{l}(q)=\frac{\sqrt{\pi}}{q^{2 r+2}} \frac{(\gamma+r) !}{\gamma !} \frac{\Gamma(\gamma+r+3 / 2)}{\Gamma(\gamma+2 r+2)} G_{\gamma}\left(\lambda+2 r, 2 r+2,1 / q^{2}\right)
$$

where $\gamma=k-r$. Except for a slight change in notation this is a generalization of a result given by Perry [10]. If one uses the second form of equation (7), and if one writes $[8$, p. 210$]$

$$
G_{n}(p, q, x)=\frac{\Gamma(q+n)}{\Gamma(p+2 n)} \sum_{r=0}^{n}(-1)^{n-k}\left(\begin{array}{l}
n \\
k
\end{array}\right) \frac{\Gamma(p+n+k)}{\Gamma(q+k)} x^{k},
$$

$\hat{F}_{l}$ becomes a sum of integrals of the form $I(\nu, a)$ as given in equation (9). Many terms vanish because of (10) and rearrangement of those remaining yields (14b) and (14d). 
4. Special cases: $\beta=1 / m, m=1,2,3, \ldots$.

Two orthogonal expansions. Here one saves a deal of calculation by exploiting the connection between the $\alpha$-curves and the $\beta$-curves through inversion in the unit circle: $((r, \theta) \rightarrow(1 / r, \theta))$. If $r_{\alpha}$ and $r_{\beta}$ are radii to $\alpha$ - and $\beta$-curves with $\alpha=\beta$, and if $d s_{\alpha}$ and $d s_{\beta}$ are elements of length of these curves then $r_{\alpha} r_{\beta}=1$ and $d s_{\alpha} / r_{\alpha}=d s_{\beta} / r_{\beta}$. Hence

$$
\begin{aligned}
& \hat{f}_{\alpha}=\int_{\alpha} f\left(r_{\alpha}, \theta\right) d s_{\alpha}=\int_{\beta=\alpha} f\left(1 / r_{\beta}, \theta\right) \frac{d s_{\beta}}{r_{\beta}^{2}}, \\
& \hat{f}_{\beta}=\int_{\beta} f\left(r_{\beta}, \theta\right) d s_{\beta}=\int_{\alpha=\beta} f\left(\frac{1}{r_{\alpha}}, \theta\right) \frac{d s_{\alpha}}{r_{\alpha}^{2}} .
\end{aligned}
$$

Applied to (13a), (13b), (14a) and (14b), these results immediately yield the following two expansions:

(i) Smooth functions of compact support in $(0,1)$.

If $\operatorname{lm}=2 r, r=0,1,2, \ldots$ and if

$$
\begin{aligned}
& F_{l}(s)=G_{k}\left(\lambda, 1 / 2, s^{2}\right), \\
& \hat{F}_{l}(q)=\text { const } x q^{2 r+1} G_{\gamma}\left(\lambda+2 r, 2 r+1, q^{2}\right)
\end{aligned}
$$

where $\gamma=k-r$ and the constant is the same as in (14b).

If $\operatorname{lm}=2 r+1, r=0,1,2, \ldots$ and if

$$
\begin{aligned}
& F_{l}(s)=s G_{k}\left(\lambda, 3 / 2, s^{2}\right), \\
& \hat{F}_{l}(q)=\text { const } x q^{2 r+2} G_{\gamma}\left(\lambda+2 r, 2 r+2, q^{2}\right)
\end{aligned}
$$

where $\gamma=k-r$ and the constant is the same as in (14d).

(ii) Smooth rapidly decreasing functions in $(1, \infty)$.

Here (13a) yields the following:

If

$$
\begin{aligned}
& F_{l}(s)=s^{-(l m+2)}\left(1-\left(1 / s^{2}\right)\right)^{\lambda-1 / 2} G_{k}\left(\operatorname{lm}+\lambda, \operatorname{lm}+1,1 / s^{2}\right) \\
& \hat{F}_{l}(q)=\text { const } x\left(1-\left(1 / q^{2}\right)\right)^{\lambda-1 / 2} C_{l m+2 k}^{\lambda}\left(1 / q^{2}\right)
\end{aligned}
$$

where the constant is the same as in (13b).

I am indebted to E. T. Quinto for discussions of a number of points in this note.

\section{REFERENCES}

1. A. M. Cormack, J. of Appl. Phys. 34 (1963), 2722.

2. __ J. of Appl. Phys. 35 (1964), 2908.

3. A. M. Cormack and E. T. Quinto, $A$ Radon transform on spheres through the origin in $R^{n}$ and applications to the Darboux equation, Trans. Amer. Math. Soc. 260 (1980), 575-581.

4. A. M. Cormack, The Radon transform on a family of curves in the plane, Proc. Amer. Math. Soc. 83 (1981), 325-330.

5. S. Helgason, The Radon transform, Birkhauser, Boston, Mass., 1980.

6. F. John, Plane waves and spherical means, Interscience, New York, 1955. 
7. A. K. Louis, Medical Image Processing Report T52, SUNY, Buffalo, 1981.

8. W. Magnus, F. Oberhettinger and R. P. Soni, Formulas and theorems for the special functions of mathematical physics, Springer-Verlag, New York, 1966.

9. R. B. Marr, On the reconstruction of a function on a circular domain from a sampling of its line integrals, J. Math. Anal. Appl. 45 (1974), 357-374.

10. R. M. Perry, On reconstructing a function on the exterior of a disk from its Radon transform, J. Math. Anal. Appl. 59 (1977), 324-341.

11. E. T. Quinto, J. Math. Anal. Appl. (to appear).

Department of Physics, Tufts University, Medford, Massachusetts 02155 